\title{
The Abandoned Side of the Internet: Hijacking Internet Resources When Domain Names Expire
}

\author{
Johann Schlamp ${ }^{1}$, Josef Gustafsson ${ }^{1}$, Matthias Wählisch ${ }^{2}$, \\ Thomas C. Schmidt ${ }^{3}$, Georg Carle ${ }^{1}$ \\ 1 Technische Universität München \\ \{schlamp, gustafss, carle\}@net.in.tum.de \\ ${ }^{2}$ Freie Universität Berlin \\ \{m.waehlisch\}@fu-berlin.de \\ 3 HAW Hamburg \\ \{schmidt\}@informatik.haw-hamburg.de
}

\begin{abstract}
The vulnerability of the Internet has been demonstrated by prominent IP prefix hijacking events. Major outages such as the China Telecom incident in 2010 stimulate speculations about malicious intentions behind such anomalies. Surprisingly, almost all discussions in the current literature assume that hijacking incidents are enabled by the lack of security mechanisms in the inter-domain routing protocol BGP.

In this paper, we discuss an attacker model that accounts for the hijacking of network ownership information stored in Regional Internet Registry (RIR) databases. We show that such threats emerge from abandoned Internet resources (e.g., IP address blocks, AS numbers). When DNS names expire, attackers gain the opportunity to take resource ownership by re-registering domain names that are referenced by corresponding RIR database objects. We argue that this kind of attack is more attractive than conventional hijacking, since the attacker can act in full anonymity on behalf of a victim. Despite corresponding incidents have been observed in the past, current detection techniques are not qualified to deal with these attacks. We show that they are feasible with very little effort, and analyze the risk potential of abandoned Internet resources for the European service region: our findings reveal that currently 73 /24 IP prefixes and 7 ASes are vulnerable to be stealthily abused. We discuss countermeasures and outline research directions towards preventive solutions.
\end{abstract}

\section{Introduction}

Internet resources today are assigned by five Regional Internet Registrars (RIRs). These non-profit organisations are responsible for resources such as blocks of IP addresses or numbers for autonomous systems (ASes). Information about the status of such resources is maintained in publicly accessible RIR databases, which are frequently used by upstream providers to verify ownership for customer 
networks. In general, networks are vulnerable to be hijacked by attackers due to the inherent lack of security mechanisms in the inter-domain routing protocol BGP. Real attacks have been observed in the past that led to the development of a variety of detection techniques and eventually of security extensions to BGP [8]11. Common to these attacks is a malicious claim of IP resources at the routing layer. However, claims of network ownership can be also made at RIR level, a fact that has received little attention so far.

In a history of more than three decades, a vast number of Internet resources have been handed out to numerous users under varying assignment policies. Some ASes or prefixes have never been actively used in the inter-domain routing, others changed or lost their original purpose when companies merged or vanished. It is not surprising that some Internet resources became abandoned, i.e. resource holders ceased to use and maintain their resources.

In this paper, we focus on threats that emerge from abandoned Internet resources. Currently, there is no mechanism that provides resource ownership validation of registered stakeholders. Instead, the control over email addresses that are stored with RIR database objects is often considered a proof of ownership for the corresponding resources. Our contribution is a generalized attacker model that takes into account these shortcomings. We thoroughly evaluate the risk potential introduced by this attack by drawing on several data sources, and show that the threat is real. Since this kind of attack enables an attacker to fully hide his identity, it makes hijacking more attractive, and significantly harder to disclose. Consequently, we show that state-of-the-art detection techniques based on network measurements are ill-suited to deal with such attacks. Even so, these attacks have been evidenced in practice, and should thus be taken into account by future research.

We continue the discussion by establishing our attacker model in Section 2 . In Section 3 we estimate the risk potential of abandoned resources, and show that there is a real threat. As a result, we outline an approach to mitigate this threat, and discuss limitations of related work in Section 4 In particular, we outline the need for a system that provides resource ownership validation. We conclude our discussion in Section 5

\section{Attacker Model}

Conventional attacks on BGP are based on its lack of origin validation, which allows an attacker to originate arbitrary prefixes or specific subnets from his own AS. We propose a new attacker model that accounts for attackers to take ownership of abandoned resources. In such a scenario, an attacker is able to act on behalf of his victim, in particular to arrange upstream connectivity. Misled upstream providers unknowingly connect one or several ASes including prefixes of the victims as instructed by an attacker who successfully hides his true identity. Following this model, the anonymous attacker can participate in the cooperative Internet exchange at arbitrary places without any formal incorrectness. In the 
following, we generalize a real incident to derive preconditions that enable this kind of attack.

\subsection{Background: The LinkTel Incident}

In previous work [17, a corresponding attack has been observed in practice, which is known as the LinkTel incident. The authors studied this attack and showed that a victim's prefixes originated from his own AS, while the victim itself abandoned his business. The authors reconstructed the attacker's course of action to claim ownership of the abandoned resources. The LinkTel incident thereby revealed a major flaw in the Internet eco-system: validation of resource ownership is most often based on manual inspection of RIR databases. In this context, it was shown that the attacker was able to gain control over the victim's DNS domain, and thus over corresponding email addresses. The involved upstream provider presumably validated that the attacker's email address was referenced by the hijacked resources' RIR database objects. Given this proof of ownership, the upstream provider was convinced by the attacker's claim to be the legitimate holder of the resources. Surprisingly, the attacker captured the victim's DNS domain by simply re-registering it after expiration.

For several months, the attacker's abuse of the hijacked resources remained unnoticed. By combining several data sources, the authors showed that the hijacked networks were utilized to send spam, to host web sites that advertised disputable products, and to engage in IRC communication. After the victim recovered his business, he learned that his networks were listed on spamming blacklists. However, the attacker's upstream provider refused to take action at first, since the victim was unable to refute the attacker's ownership claims.

\subsection{Preconditions for an Attack}

Based on the insights gained from the LinkTel incident, we show that the attacker's approach can be generalized. To enable hijacking of Internet resources, the following preconditions have to be met: (a) Internet resources are evidentially abandoned and (b) the original resource holder can be impersonated.

If an organisation goes out of business in an unsorted manner, these conditions are eventually met. As a first consequence, the organisation ceases to use and maintain its resources. If this situation lasts over a longer period of time, the organisation's domain name(s) expire. Since day-to-day business lies idle, re-registration and thus impersonation becomes practicable for an attacker. At that moment, upstream connectivity can be arranged on behalf of the victim, since face-to-face communication is not required in general. Routers can be sent via postal service, or even be rented on a virtualized basis. Details on BGP and network configuration are usually exchanged via email, IRC, or cellular phone, and payment can be arranged anonymously by bank deposits or other suitable

payment instruments. Without revealing any evidence about his real identity, the attacker is able to stealthily hijack and deploy the abandoned resources. 


\subsection{Implications}

The implications of this attacker model are manifold. First, an attacker may act on behalf of a victim, thereby effectively hiding his own identity and impeding disclosure. This makes hijacking more attractive as it enables riskless network abuse. It hinders criminal prosecution, and could be used to deliberately create tensions between organisations or even countries. Due to the lack of a system for resource ownership validation, these attacks only depend on idle organisations or missing care by legal successors of terminated businesses. Even after the discovery of such an attack, it is difficult for the victim to mitigate since reclaiming ownership is the word of one person against another at first. The LinkTel incident [17] proves that this is not only a realistic scenario: such attacks are actually carried out in practice.

The benefit of attacks based on abandoned resources can even be higher than in the case of conventional attacks. Hijacking productive networks rarely lasts for more than a few hours, since the victim can receive great support in mitigating the attack. Moreover, for most cases, the benefit is reduced to blackholing a victim's network - with the Youtube-Pakistan incident being a prominent example. In addition, monitoring systems for network operators exist that raise alarms for unexpected announcements of their prefixes. However, due to the very nature of abandoned resources, virtually no one is going to take notice of an attack. Our attacker model thus accounts for stealthily operating attackers who aim at persistently maintaining malicious services.

\section{Abandoned Internet Resources}

We identify readily hijackable Internet resources by searching RIR databases for unmaintained resource objects. Subsequently, we distinguish between resources that are still in use, with potential for network disruption, and resources that are fully abandoned and ready to be abused stealthily. Such resources are especially attractive for attackers for two reasons. First, the resource is assigned to an organisation for operational use and thus represents a valid resource in the Internet routing system. Second, an attacker can easily claim ownership by taking control of the contact address referenced by corresponding RIR database objects, iëby re-registering a domain name.

Consequently, we look for RIR database objects that reference email addresses with expired DNS names. Since the inference of invalid domain names can also be the result of poorly maintained resource objects or typing errors, it is important to take into account recent database activities for individual resource owners, and to correlate this information with BGP activity.

The following analysis is based on archived RIPE database snapshots over 2.5 years (23 February, 2012 till 9 July, 2014). Our results are representative for the European service region only, but similar analyses can be done with little

effort for other service regions, too. 


\begin{tabular}{lrrr}
\hline Object type & Frequency & \multicolumn{2}{c}{ DNS references } \\
\hline inetnum & $3,876,883$ & $1,350,537$ & $(34.84 \%)$ \\
domain & 658,689 & 97,557 & $(14.81 \%)$ \\
route & 237,370 & 50,300 & $(21.19 \%)$ \\
inet6num & 231,355 & 8,717 & $(3.77 \%)$ \\
organisation & 82,512 & 0 & $(0.00 \%)$ \\
mntner & 48,802 & 0 & $(0.00 \%)$ \\
aut-num & 27,683 & 6,838 & $(24.70 \%)$ \\
role & 20,684 & 14,430 & $(69.76 \%)$ \\
as-set & 13,655 & 2,500 & $(18.31 \%)$ \\
route6 & 9,660 & 723 & $(7.48 \%)$ \\
irt & 321 & 162 & $(50.47 \%)$ \\
\hline Total & $\mathbf{5 , 2 3 9 , 2 0 1}$ & $\mathbf{1 , 5 3 1 , 7 6 4}$ & $\mathbf{( 2 9 . 2 4 \% )}$ \\
\hline
\end{tabular}

Table 1. Data objects stored in the RIPE database, and references to DNS names. 9 July, 2014.

\subsection{Resource Candidates from RIR Database}

RIPE, like all other RIRs, provides publicly available database snapshots on a daily basis. Most of the personally related information is removed due to privacy concerns. Some attributes, however, remain unanonymized, which we utilize to extract DNS names.

Available Data Objects The RIPE database holds more than 5.2 million objects. These objects can be updated from the Web or via email. Most of these objects optionally hold an email address in the notify field, to which corresponding update notifications are sent. Despite anonymization, we found that these notify fields are preserved in the publicly available database snapshots, which is also the case for abuse-mailbox attributes. To extract DNS names, we parse these email addresses where applicable.

Table 1 shows the distribution of stored objects by type along with the number of DNS names we were able to extract. Although we found more than 1.5 million references to DNS names, the total number of distinct names is only 21,061 . This implies that, on average, more than 72 objects reference the same DNS name. The overall fraction of objects that reference a domain name is $29.24 \%$, which is surprisingly high since the database snapshots are considered to be anonymized.

Hijackable Internet resources are given by inetnum and aut-num objects, which represent blocks of IP addresses and unique numbers for autonomous systems respectively. Exemplary database objects are provided in Figure 1, further details on the RIPE database model and update procedures are available at [16].

It is worth noting that the attacker neither needs authenticated access to the database nor does the attacker need to change the database objects. The 


$\begin{array}{ll}\text { inetnum: } & \text { 194.28.196.0 - 194.28.199.255 } \\ \text { netname: } & \text { UA-VELES } \\ \text { descr: } & \text { LLC "Unlimited Telecom" } \\ \text { descr: } & \text { Kyiv } \\ \text { notify: } & \text { internet@veles-isp.com.ua } \\ \text { mnt-by: } & \text { VELES-MNT } \\ & \\ \text { aut-num: } & \text { AS51016 } \\ \text { as-name: } & \text { VALES } \\ \text { descr: } & \text { LLC "Unlimited Telecom" } \\ \text { notify: } & \text { internet@veles-isp.com.ua } \\ \text { mnt-by: } & \text { VELES-MNT }\end{array}$

Fig. 1. Examples of RIPE database objects (inetnum and aut-num objects).

attacker only needs to derive a valid contact point. We assume that the (publicly available) notification address usually belongs to the same DNS domain as the technical contact point. Detailed analysis is subject to future work; in our study, we disregard groups of objects that reference more than a single DNS domain as a precaution.

Grouping Objects by Maintainer The RIPE database is mostly maintained by resource holders themselves. Its security model is based on references to mntner (maintainer) objects, which grant update and delete privileges to the person holding a mntner object's password. This security model allows us to infer objects under control of the same authority by grouping objects with references to a common mntner object. We use these maintainer groups to estimate the impact of an attack for individual authorities: On average, we observed nearly 110 such references per mntner object, with a maximum of up to 436,558 references 4 . The distribution of the number of objects per maintainer group is presented in Figure 2.

For each of the maintainer groups, we obtain the set of all DNS names referenced by a group's objects. To unambiguously identify maintainer groups with expired domains, we merge disjoint groups that reference the same DNS domain, and discard groups with references to more than one DNS name. From an initial amount of 48,802 maintainer groups, we discard (a) 937 groups of zero size, i.e. unreferenced mntner objects, (b) 31,586 groups without domain name references, and (c) 4,990 groups with multiple references. The remaining 11,289 groups can be merged to 8,441 groups by identical DNS names. We further discard groups that do not include any hijackable resources, i.e. inetnum and aut-num objects, which finally leads us to 7,907 object groups.

Note that the number of these groups is a lower bound: an attacker could identify even more with access to unanonymized RIPE data. As discussed above,

${ }^{4}$ The meta information refers to Interbusiness Network Administration Staff of Telecom Italia. 


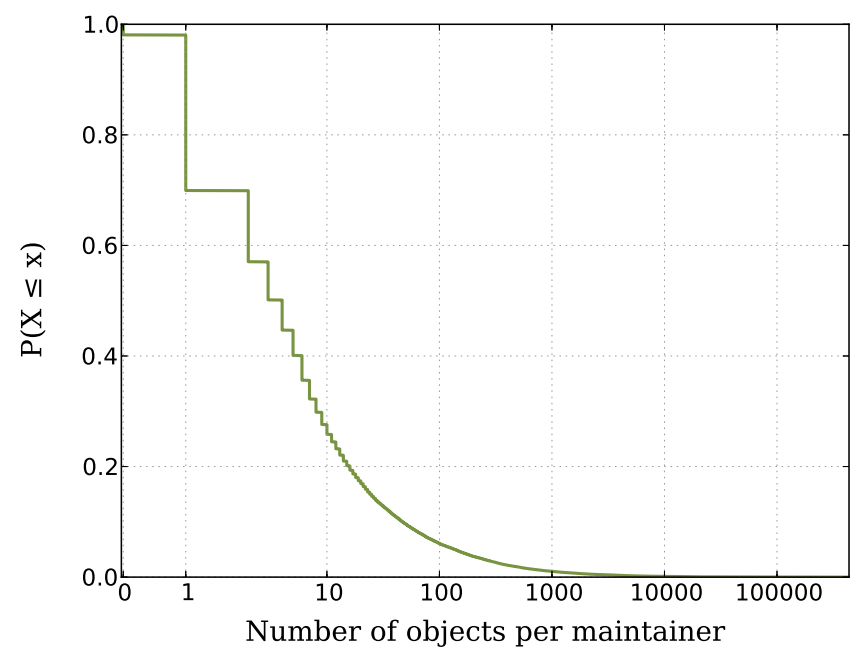

Fig. 2. RIPE database objects grouped by references to a common maintainer object $(\mathrm{CCDF})$.

each of these groups is maintained by a single entity. If a group's DNS name expires, we consider the entity's resources to be a valuable target for an attacker.

\subsection{Refinement by Activity Measures}

To confirm that a set of resources is abandoned, our approach is based on complementary data sources. We start with domain names that expire, which is a strong yet inconclusive indication for a fading resource holder. We gain further evidence by considering only resources that are neither changed in the RIPE database nor announced in BGP. Including both administrative (DNS, RIPE) and an operational (BGP) measures gives a comprehensive picture on the utilization of the resources.

Lifetime of Domain Names We used the whois system to query expiry dates for all extracted DNS names (cf., Section 3.1). Figure 3 shows the distribution of these dates. At the time of writing, 214 domain names have been expired. Another 121 names expire within the week, given that the owners miss to renew their contracts. The most frequent top level domains are .com $(27.9 \%)$, .$r u(21.5 \%)$, and .net (13.0\%), while the most frequent expired TLDs are .ru $(20.1 \%)$, it $(16.4 \%)$, and .com $(9.81 \%)$. The longest valid domains are registered until 2108 and mostly represent governmental institutions. The longest expired domain has been unregistered for nearly 14 years. With respect to the maintainer groups derived above, a total of 65 groups that reference expired DNS names remain. These groups hold 773 /24 networks and 54 ASes, and are subject to closer investigation. 


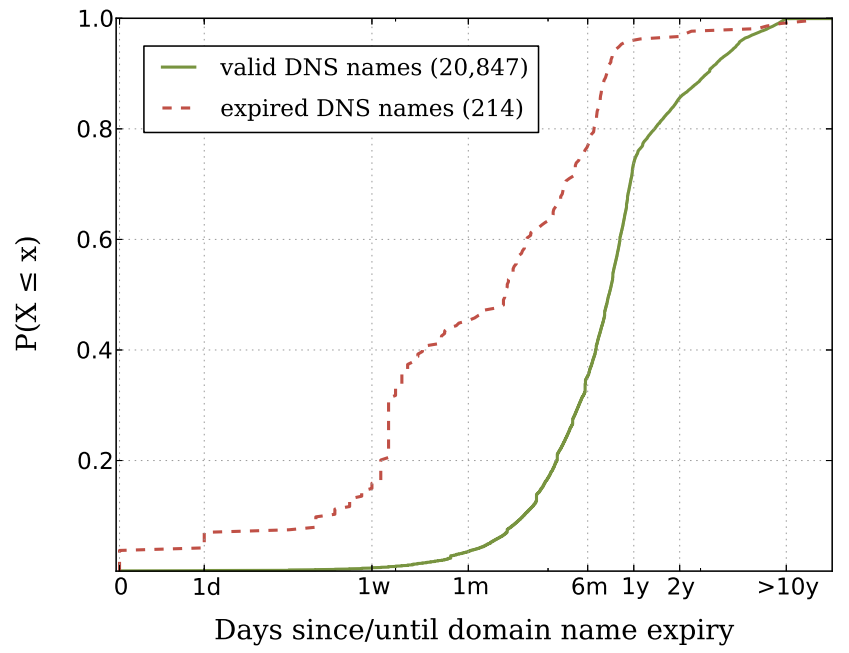

Fig. 3. Expiry dates for DNS names referenced by RIPE database objects.

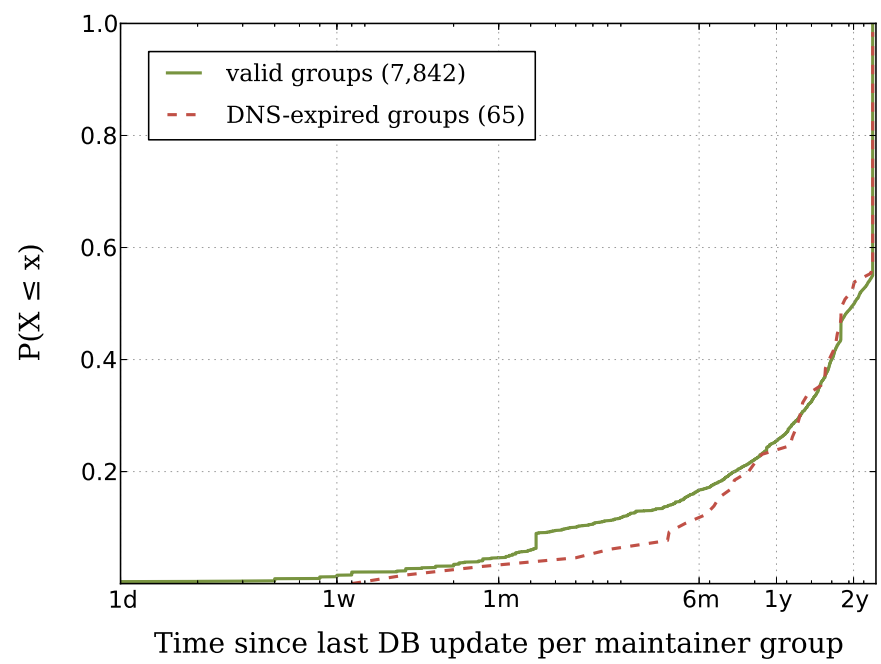

Fig. 4. RIPE database updates by maintainer group (CDF).

RIPE Database Updates For each of the 7,907 maintainer groups - divided into 7,842 valid groups and 65 with expired DNS names - we extracted the minimum time since the last change for any of its database objects. Note that we filtered out automated bulk updates that affected all objects of a certain typ 65 . Figure 4 shows the distribution of database updates for groups with valid and for groups with expired domain names. While about $10 \%$ of the valid groups

${ }^{5}$ For instance, RIPE added a new status attribute to all aut-num objects on 27 May, 2014. 
show changes within two months, DNS-expired groups differ strikingly: the 10\%quantile is at nearly 5 months. Hence, given these long times without updates, we consider resource groups that exhibit an object update within 6 months to be still maintained and not abandoned. Note that we do not assume inactivity in absence of such changes.

BGP Activity To confirm inactivity, we correlate the RIPE database updates with activities in the global routing system. For that, we analyze all available BGP update messages from the RouteViews Oregon's feed for the same time frame. This data set comprises 83,255 files with 18.4 billion announcements and 1.04 billion withdraw messages for resources assigned by RIPE. Given this data, we are able to extract two indicators: (1) the time since an IP prefix was last visible from the RouteViews monitor, and (2) the time since the last deployment of a RIPE-registered $A S$ number by looking at AS path attributes. Figure 5 shows the distribution of last activity in BGP for any Internet resource in our maintainer groups. Nearly $90 \%$ of resources in valid groups are visible in BGP at the moment. Surprisingly, most of the remaining groups did not show any activity at all during the last 2.5 years. About $75 \%$ of the DNS-expired resources are present in today's routing table - and are thus still actively used. The remaining resources did show some activity in the past $(10 \%)$ or were never observed in BGP during our analysis period (15\%).

These findings confirm our assumption that inactivity in the RIPE database does not necessarily imply operational shutdown. While up to $85 \%$ of the expired resources were seen in BGP within the last 2.5 years, Figure 4 indicates that not more than $55 \%$ of the expired resources received an update in the RIPE database. We further learn that some expired resources did show BGP activity in the past, and do not show any activity today. Note that we disregard resources with recent BGP activity. These resources could potentially be hijacked already; however, attacks that started before our analysis are beyond the scope of our approach.

\subsection{Hijackable Resources}

So far, we learned that 65 maintainer groups with a total of 773 /24 networks and 54 ASes reference expired DNS names. Our activity measures further indicate that valid groups yield higher activity than expired groups. By combining these measures, we are able to infer resources that are inactive from both an administrative and an operational point of view. Figure 6 shows the time since the latest change by any of these measures, i.e., the minimum value of both measures.

This combined activity measure clearly splits the 65 expired maintainer groups into two disjoint sets: 52 cases were active within the last 3 months, while 13 cases did not show any activity for more than 6 months. We consider these remaining 13 cases to be effectively abandoned. These resource groups represent a total number of 15 inetnum objects (with an equivalent of 73 /24 networks) and 7 aut-num (i.e., AS number) objects. 


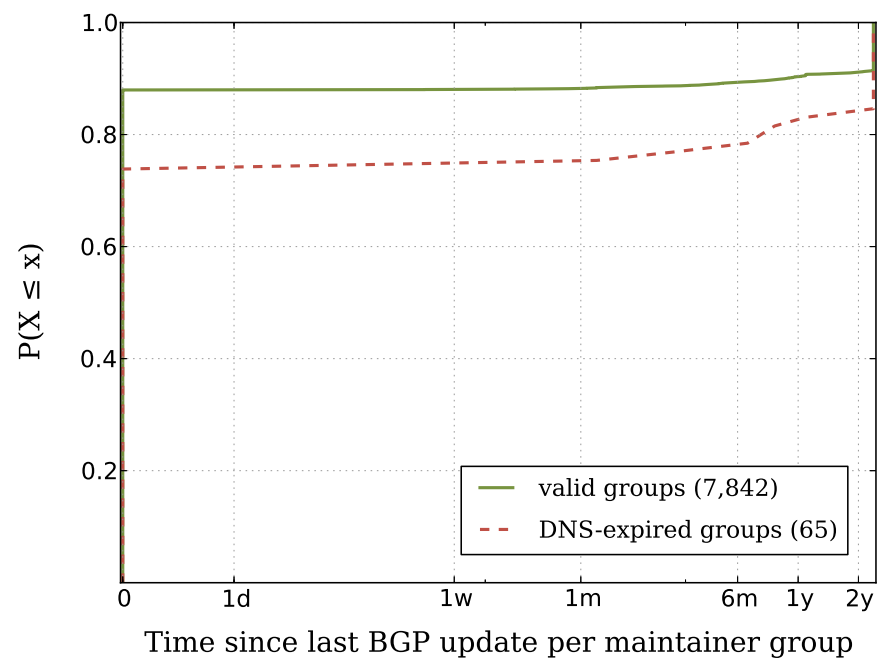

Fig. 5. BGP update messages observed by maintainer group (CDF).

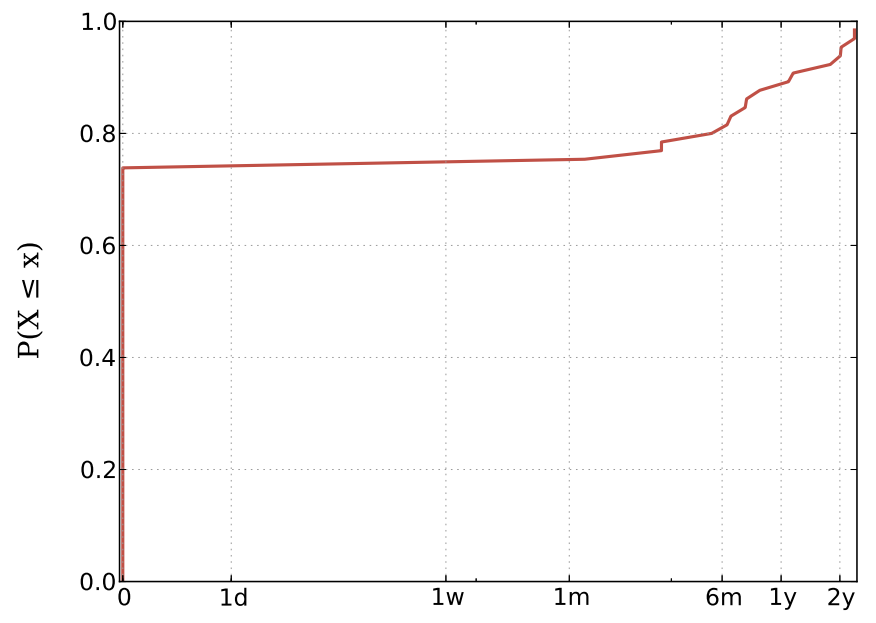

Time since last RIPE/BGP update per maintainer group

Fig. 6. Combined RIPE/BGP activity by maintainer group (CDF).

Now that we have identified vulnerable resources, we feel obliged to protect these resources. Since any attacker could repeat our analysis, we are going to contact endangered resource holders before publishing our findings. Although communication via e-mail is futile due to expired domains, we can fall back on telephone numbers provided in the RIPE database to reach out for the operators. 


\section{Research Agenda}

For the problem of abandoned Internet resources, one might argue that the threat is not caused by a technical but a social problem because operators agree to their peering relations based on a weak authentication scheme. This scheme can be replaced by stronger verification - the required data already exists. RIRs have contracts with the owners of delegated resources and thus are aware of more reliable contact points (e.g., telephone numbers). However, the current situation shows that we need mechanisms, tools, and procedures which are not tedious for operators but allow for easy resource verification. Our approach to identify abandoned resources can be easily extended to continuously monitor resources of all RIRs. This would allow us to warn network operators about potential risks. Finding scalable approaches to implement early warning and prevention in real-time, though, is an open research issue.

\subsection{Limitations of Related Work}

Current research is particularly focused on the detection of BGP hijacking attacks. Proposed mitigation techniques look on the control plane, the data plane, or both. Control plane monitoring is used to identify anomalies in BGP routing tables to infer attacks 9114216]. Such approaches are prone to false positives due to legitimate causes for anomalies. Techniques based on data plane measurements account for changes of the router topology [23|22], or of hosts in supposedly hijacked networks [51418. These approaches rely on measurements carried out before and during an attack. Beyond that, studies on the malicious intent behind hijacking attacks exist [15]19]17|20.

All detection approaches require the observation of suspicious routing changes. Attacks based on our attacker model take place outside the routing system, and thus do not lead to noticeable routing changes - apart from a supposedly legitimized organisation starting to reuse its Internet resources. Hence, current detection systems are incapable to deal with this kind of attack.

The DNS has been widely studied in the context of malicious network activities, mainly concerning spammers or fraud websites. Proactive blacklisting of domain names [3] does not help in our scenario as the threat is effective on the routing layer. Identifying orphaned DNS servers [7] is also out of scope of this paper as the attacker does not leverage the DNS server but the expiring domain.

\subsection{Resource Ownership Validation}

Despite its effectiveness, we consider our approach to detect and monitor abandoned resources as outlined above an intermediate solution only. In fact, we argue that there is a need for resource ownership validation.

There is ongoing effort to increase the deployment of a Resource Public Key Infrastructure (RPKI) [11. In its present state, the RPKI allows for validation of route origins by using cryptographically secured bindings between AS numbers and IP prefixes. This mechanism prevents common hijacking attacks. In terms of 
hijacking abandoned resources, however, this system is ineffective in its current form since the abandoned origin AS is taken over as well, and origin validation performed by BGP routers [13] will indicate a valid BGP update.

Even though the RPKI itself can be misused [2, at the moment it represents the only mechanism for proofing securely ownership of Internet resources. We merely lack a clear procedure in the context of abandoned Internet resources. One approach could be the following operational rule: a peering request is only established when resource objects of the requesting peer exist in the RPKI. Recent time stamps for these objects indicate that the requesting peer has control over the resources as only authorized users can create such objects. Such a scheme seems feasible from the operational perspective and might even increase the incentives to deploy RPKI.

RPKI is part of BGPsec, an even larger effort to secure BGP. This extension to the protocol remedies the risk of hijacking abandoned resources due to its path validation capabilities: in our model, an attacker cannot provide valid cryptographic keys to sign update messages as specified by BGPsec [10]. However, the development of BGPsec is at an early stage, and the benefit compared to pure origin validation is questionable in particular in sparse deployment scenarios [12.

Future research should be carried out on enabling Internet service providers to validate resource ownership of customers. We see the potential of such a system not only in preventing attackers from hijacking abandoned Internet resources. It would also establish trust in customer-provider and peer-to-peer relationships, as well as in resource transfers issued by RIRs or LIRs.

\section{Conclusion}

Motivated by a real-world case study, we introduced a generalized attacker model that is aimed on the hijacking of abandoned Internet resources. We showed that such an attack is feasible with little effort, and effectively hides the attacker's identity by acting on behalf of a victim. By studying orthogonal data sources over a period of more than 30 months, we could give evidence of a high risk potential of such attacks. Only in the European RIR database, we found 214 expired domain names that control a total of $773 / 24$ networks and 54 ASes, all of which can be easily hijacked. About $90 \%$ of these resources are still in use, which enables an attacker to disrupt operational networks. The remaining $10 \%$ of the resources are fully abandoned, and ready to be stealthily abused.

Our findings led us to the conclusion that state-of-the-art systems are limited to deal with this kind of attack. More importantly, we argued that there is a need for resource origin validation. Such a framework would not only prevent attacks, but could also strengthen today's Internet eco-system by establishing trust in resource ownership. 
Ethical Considerations In this paper, we sketched a new attack vector. Up until now, it is unclear how common such attacks are; our findings thus might trigger new malicious activities. However, we also showed that this attack is already known to attackers, and we sketched countermeasures to mitigate this concern. In addition, we contact the holders of vulnerable resources before publication of our findings.

Acknowledgements This work has been supported by the German Federal Ministry of Education and Research (BMBF) under support code 01BY1203C, project Peeroskop, and by the European Commission under the FP7 project EINS, grant number 288021.

\section{References}

1. H. Ballani, P. Francis, and X. Zhang. A study of prefix hijacking and interception in the Internet. In Proc. ACM SIGCOMM 200\%, pages 265-276, 2007.

2. D. Cooper, E. Heilman, K. Brogle, L. Reyzin, and S. Goldberg. On the Risk of Misbehaving RPKI Authorities. In Proc. of HotNets-XII, New York, NY, USA, 2013. ACM.

3. M. Felegyhazi, C. Kreibich, and V. Paxson. On the Potential of Proactive Domain Blacklisting. In Proc. of the 3rd USENIX LEET Conference, Berkeley, CA, USA, 2010. USENIX Association.

4. Hong, Seong-Cheol and Ju, Hong-Taek and Hong, James W. IP Prefix Hijacking Detection Using Idle Scan. In 12th Asia-Pacific Network Operations and Management Conference (APNOMS'09), pages 395-404, 2009.

5. X. Hu and Z. M. Mao. Accurate real-time identification of IP prefix hijacking. In Proc. IEEE Symposium on Security and Privacy, pages 3-17, 2007.

6. Q. Jacquemart, G. Urvoy Keller, and E. Biersack. A longitudinal study of BGP MOAS prefixes. In 6th Int. Workshop on Traffic Monitoring and Analysis, (TMA '14), 2014.

7. A. J. Kalafut, M. Gupta, C. A. Cole, L. Chen, and N. E. Myers. An Empirical Study of Orphan DNS Servers in the Internet. In Proc. of the 10th ACM SIGCOMM IMC, pages 308-314, New York, NY, USA, 2010. ACM.

8. S. Kent, C. Lynn, and K. Seo. Secure Border Gateway Protocol (SBGP). IEEE Journal on Selected Areas in Communications, 18(4), April 2000.

9. M. Lad, D. Massey, D. Pei, Y. Wu, B. Zhang, and L. Zhang. PHAS: A prefix hijack alert system. In Proc. 15th USENIX Security Symposium, volume 15, 2006.

10. M. Lepinski. BGPSEC Protocol Specification. Internet-Draft - work in progress 00, IETF, March 2011.

11. M. Lepinski and S. Kent. An Infrastructure to Support Secure Internet Routing. RFC 6480, IETF, February 2012.

12. R. Lychev, S. Goldberg, and M. Schapira. Bgp security in partial deployment: Is the juice worth the squeeze? In Proc. of ACM SIGCOMM, pages 171-182, New York, NY, USA, 2013. ACM.

13. P. Mohapatra, J. Scudder, D. Ward, R. Bush, and R. Austein. BGP Prefix Origin Validation. RFC 6811, IETF, January 2013.

14. J. Qiu and L. Gao. Detecting bogus BGP route information: going beyond prefix hijacking. In In Proc. 3rd Int. Conf. on Security and Privacy in Communication Networks (SecureComm), 2007. 
15. A. Ramachandran and N. Feamster. Understanding the network-level behavior of spammers. In Proc. ACM SIGCOMM 2006, 2006.

16. RIPE NCC. RIPE Database Update Reference Manual. http://www.ripe.net/ data-tools/support/documentation/RIPEDatabaseUpdateManual20140425_ edit.pdf.

17. J. Schlamp, G. Carle, and E. W. Biersack. A forensic case study on as hijacking: the attacker's perspective. ACM SIGCOMM CCR, 43(2):5-12, 2013.

18. X. Shi, Y. Xiang, Z. Wang, X. Yin, and J. Wu. Detecting prefix hijackings in the Internet with argus. In Proc. ACM SIGCOMM Internet Measurement Conference $(I M C), 2012$.

19. P.-A. Vervier and O. Thonnard. SpamTracer: How stealthy are spammers? In 5th Int. Workshop on Traffic Monitoring and Analysis, (TMA '13), 2013.

20. Vervier, Pierre-Antoine and Jacquemart, Quentin and Schlamp, Johann and Thonnard, Olivier and Carle, Georg and Urvoy-Keller, Guillaume and Biersack, Ernst W. and Dacier, Marc. Malicious BGP Hijacks: Appearances can be deceiving. In IEEE ICC Communications and Information Systems Security Symposium (ICC CISS 2014), 2014.

21. M. Wählisch, O. Maennel, and T. C. Schmidt. Towards Detecting BGP Route Hijacking Using the RPKI. ACM SIGCOMM CCR, 42(4):103-104, August 2012.

22. Z. Zhang, Y. Zhang, Y. C. Hu, Z. M. Mao, and R. Bush. iSPY: Detecting IP prefix hijacking on my own. IEEE/ACM Trans. on Networking, 18(6):1815-1828, 2010.

23. C. Zheng, L. Ji, D. Pei, J. Wang, and P. Francis. A light-weight distributed scheme for detecting IP prefix hijacks in real-time. In Proc. ACM SIGCOMM 200\%, pages 277-288, 2007. 